\title{
Scaling-up vaccine production: implementation aspects of a biomass growth observer and controller
}

\author{
Zita I. T. A. Soons $\cdot$ Jan van den IJssel • \\ Leo A. van der Pol · Gerrit van Straten • \\ Anton J. B. van Boxtel
}

Received: 24 March 2008 / Accepted: 12 July 2008/Published online: 31 July 2008

(C) The Author(s) 2008

\begin{abstract}
This study considers two aspects of the implementation of a biomass growth observer and specific growth rate controller in scale-up from small- to pilot-scale bioreactors towards a feasible bulk production process for whole-cell vaccine against whooping cough. The first is the calculation of the oxygen uptake rate, the starting point for online monitoring and control of biomass growth, taking into account the dynamics in the gas-phase. Mixing effects and delays are caused by amongst others the headspace and tubing to the analyzer. These gas phase dynamics are modelled using knowledge of the system in order to reconstruct oxygen consumption. The second aspect is to evaluate performance of the monitoring and control system with the required modifications of the oxygen consumption calculation on pilot-scale. In pilot-scale fed-batch cultivation good monitoring and control performance is obtained enabling a doubled concentration of bulk vaccine compared to standard batch production.
\end{abstract}

Keywords Scale-up · Monitoring and control .

Bordetella pertussis · Gas-phase dynamics · Fed-batch .

Oxygen uptake rate

\section{List of symbols}

$a, b, c, d \quad$ constants for dual substrate model of B. pertussis

Z. I. T. A. Soons · G. van Straten · A. J. B. van Boxtel ( $₫)$

Systems and Control Group, Wageningen University,

P. O. Box 17, 6700 AA Wageningen, The Netherlands

e-mail: ton.vanboxtel@wur.nl

Z. I. T. A. Soons - J. van den IJssel - L. A. van der Pol Unit Research and Development, Netherlands Vaccine Institute,

P. O. Box 457, 3720 AL Bilthoven, The Netherlands

e-mail: zita.soons@gmail.com

\begin{tabular}{|c|c|}
\hline$C_{\mathrm{A}}$ & acetoacetate concentration $\left(\mathrm{mmol} \mathrm{l}^{-1}\right)$ \\
\hline$C_{\mathrm{G}}, C_{\mathrm{L}}$ & $\begin{array}{l}\text { glutamate and lactate concentration } \\
\text { respectively }\left(\mathrm{mmol} \mathrm{l}^{-1}\right)\end{array}$ \\
\hline$C_{\mathrm{X}}$ & biomass concentration $\left(\mathrm{OD}_{590}\right)$ \\
\hline$C_{\mathrm{O}}^{\mathrm{L}}$ & $\begin{array}{l}\text { oxygen concentration in bioreactor } \\
\left(\mathrm{mmol} \mathrm{OD}^{-1} 1^{-1}\right)\end{array}$ \\
\hline$C_{\mathrm{O}}^{*}$ & $\begin{array}{l}\text { oxygen concentration at gas-liquid } \\
\text { interface }\left(\mathrm{mmol} \mathrm{OD}^{-1} \mathrm{l}^{-1}\right)\end{array}$ \\
\hline CSTR & continuous stirred tank reactor \\
\hline E & mean absolute error $\left(\mathrm{h}^{-1}\right)$ \\
\hline$F_{\text {calc }}$ & feed rate by prior calculation $\left(1 \mathrm{~h}^{-1}\right)$ \\
\hline$F_{\mathrm{G}}$ & gas flow rate $\left(1 \mathrm{~h}^{-1}\right)$ \\
\hline$F_{\mathrm{G}+\mathrm{L}}$ & $\begin{array}{l}\text { substrate feed rate (glutamate + lactate) } \\
\left(1 \mathrm{~h}^{-1}\right)\end{array}$ \\
\hline$F_{\mathrm{I}}, F_{\mathrm{P}}$ & $\begin{array}{l}\text { integral, proportional action feed } \\
\text { rate }\left(1 \mathrm{~h}^{-1}\right)\end{array}$ \\
\hline$H$ & Henry coefficient $\left(\mathrm{Pa} 1 \mathrm{mmol}^{-1}\right)$ \\
\hline$k_{\mathrm{L}} a$ & oxygen transfer coefficient $\left(\mathrm{h}^{-1}\right)$ \\
\hline$m_{\mathrm{O}}$ & $\begin{array}{l}\text { maintenance coefficient on oxygen } \\
\left(\mathrm{mmol} \mathrm{OD}^{-1} \mathrm{~h}^{-1}\right)\end{array}$ \\
\hline$N$ & length of moving window \\
\hline$O$ & oscillation measure $\left(\mathrm{h}^{-1}\right)$ \\
\hline $\mathrm{O}_{2}$ & oxygen fraction $(-)$ \\
\hline OD & optical density at $590 \mathrm{~nm}$ \\
\hline OTR, OUR & $\begin{array}{l}\text { oxygen transfer rate, oxygen uptake rate } \\
\left(\mathrm{mmol} \mathrm{l}^{-1} \mathrm{~h}^{-1}\right)\end{array}$ \\
\hline$p$ & pressure $(\mathrm{Pa})$ \\
\hline$R$ & gas constant $\left(\mathrm{J} \mathrm{mmol}^{-1} \mathrm{~K}^{-1}\right)$ \\
\hline$t$ & cultivation time $(\mathrm{h})$ \\
\hline$T$ & sampling time $(\mathrm{h})$ \\
\hline$T_{\mathrm{D}}$ & transport delay $(\mathrm{h})$ \\
\hline$T_{\mathrm{L}}$ & temperature in bioreactor $(\mathrm{K})$ \\
\hline$u$ & inputs \\
\hline$V$ & liquid volume (l) \\
\hline$V_{\text {tot }}$ & volume total gas-phase (1) \\
\hline
\end{tabular}

$c_{\mathrm{A}}$

$C_{\mathrm{G}}, C_{\mathrm{L}}$

$C_{\mathrm{X}}$

$C_{\mathrm{O}}^{\mathrm{L}}$

$C_{\mathrm{O}}^{*}$

CSTR

E

$F_{\text {cal }}$

$F_{\mathrm{G}}$

$F_{\mathrm{I}}, F_{\mathrm{P}}$

$H$

$k_{\mathrm{L}} a$

$m_{\mathrm{O}}$

$N$

$O$

$\mathrm{O}_{2}$

OD acetoacetate concentration $\left(\mathrm{mmol} \mathrm{l}^{-1}\right)$

respectively $\left(\mathrm{mmol} \mathrm{l}^{-1}\right)$

$\left(\mathrm{mmol} \mathrm{OD} \mathrm{OD}^{-1} \mathrm{l}^{-1}\right)$

oxygen concentration at gas-liquid $D^{-1} 1^{-1}$

mean absolute error $\left(\mathrm{h}^{-1}\right)$

feed rate by prior calculation $\left(1 \mathrm{~h}^{-1}\right)$

$\left(1 \mathrm{~h}^{-1}\right)$

thegral, proportional action feed

Henry coefficient $\left(\mathrm{Pa} 1 \mathrm{mmol}^{-1}\right)$

nt onygen

mmol OD $\left.{ }^{-1} h^{-1}\right)$

oxygen fraction $(-)$

$\left(\mathrm{mmol} \mathrm{l} \mathrm{l}^{-1} \mathrm{~h}^{-1}\right.$ )

pressure $(\mathrm{Pa})$

cultivation time $(\mathrm{h})$

sampling time $(\mathrm{h})$

transport delay $(\mathrm{h})$

temperature in bioreactor $(\mathrm{K})$

liquid volume (l)

volume total gas-phase (1) 


$$
\begin{array}{ll}
V_{\text {tubing }} & \text { volume tubing }(\mathrm{l}) \\
x, y & \text { states, outputs } \\
Y_{\mathrm{O}} & \text { biomass yield on oxygen }\left(\mathrm{OD} \mathrm{mmol}^{-1}\right) \\
\varepsilon & \text { error between set point and process } \\
& \mu_{\text {set }}-\hat{\mu}\left(\mathrm{h}^{-1}\right)
\end{array}
$$

\section{Greek symbols}

$\beta \quad$ adaptation rate

$\gamma_{1}, \gamma_{2}$ tuning parameters for $\mu$ control

$\mu \quad$ specific growth rate $\left(\mathrm{h}^{-1}\right)$

$\begin{array}{ll}\text { Superscripts and subscripts } \\ \wedge \text {,EKF } & \text { observed values } \\ \mathrm{a} & \text { auxiliary } \\ \text { in } & \text { inlet } \\ \mathrm{m} & \text { measured (in outlet stream) } \\ \mathrm{mix} & \text { outlet stream incl. mixing effect in the total } \\ & \text { gas phase } \\ \text { out } & \text { outlet stream of the bioreactor } \\ \text { set } & \text { set-point } \\ \mathrm{p} & \text { predicted values }\end{array}$

\section{Introduction}

Currently, most biopharmaceuticals are produced in a batch or fed-batch cultivation. The quality of the product is formed in this step and is the result of the metabolic state of the micro-organisms. It is therefore essential to measure and control the metabolic state of the process. Metabolic activity is difficult to measure directly due to the lack of sensors, but respiration can be monitored by the oxygen mass balance. The oxygen uptake rate can in turn be used to reconstruct the specific growth rate and biomass using an observer.

Monitoring and control systems in biotechnology are usually designed and tested in laboratory-scale experiments. Its application on production-scale is limited in literature and is not that straightforward in practice. In scaling-up from laboratory to pilot-scale and beyond two issues were encountered for which solutions have been worked out in this paper. The first issue is the calculation for oxygen consumption on large-scale, the starting point for online monitoring and control, taking into account the dynamics in the gas-phase. The second issue is the actual application of the observer and controller for biomass growth to pilot-scale cultivation. Changing from small to medium- or large-scale systems usually requires re-tuning of the controller settings. The applied adaptive controller was designed such that its settings are the result of the process characteristics [1] and it automatically tunes its settings if performance is poor [2]. In this work, we investigated the properties and performance of the specific growth rate controller in scale-up from laboratory to pilotscale.

In the application for whooping cough vaccine production, the oxygen uptake rate was the input for the monitoring and control system. The oxygen uptake rate for small-scale systems is usually calculated using Eq. 1, e.g., [3]:

$\mathrm{OUR} \approx \mathrm{OTR}=k_{\mathrm{L}} a\left(C_{\mathrm{O}}^{*}-C_{\mathrm{O}}^{\mathrm{L}}\right)$

where $C_{\mathrm{O}}^{*}$ and $C_{\mathrm{O}}^{\mathrm{L}}$ are the oxygen concentrations at the gasliquid interface and in the cultivation broth, respectively. This method of OUR calculation may, however, be inappropriate for pilot- and large-scale systems, because the bioreactor may not be ideally mixed and the oxygen transfer coefficient $k_{\mathrm{L}} a$ is often not accurately known on the large-scale.

Other approaches to retrieve the oxygen uptake rate require switching of the aeration gas composition, e.g., [4], and thus introducing systematic disturbances. Such probing should be avoided in cultivation systems for biopharmaceutical production as it may affect critical variables. Furthermore, the approach may be less suited for dynamic systems in the view of online monitoring and control, where frequent oxygen consumption "measurements" are necessary.

An alternative that eliminates the need to know $k_{\mathrm{L}} a$ is the computation using off-gas measurements. Often the computation of OUR using off-gas measurements is based on a steady-state assumption (for example in [5-12]):

$\mathrm{OUR} \approx \mathrm{OTR}=\frac{F_{\mathrm{G}}}{V}\left(\mathrm{O}_{2}^{\text {in }}-\mathrm{O}_{2}^{\mathrm{m}}\right) \frac{p}{R T_{\mathrm{L}}}$

where $F_{\mathrm{G}}$ is the gas flow rate, $\mathrm{O}_{2}^{\text {in }}$ the oxygen fraction in the inlet stream, $\mathrm{O}_{2}^{\mathrm{m}}$ the measured oxygen fraction in the outlet stream, $p$ is the pressure in the bioreactor, $R$ the gas constant, and $T_{\mathrm{L}}$ the temperature in the bioreactor. These offgas fractions, however, do not reflect the actual concentrations in the bioreactor under dynamic conditions [13]. This is due to the presence of a headspace and tubing, which cause additional dynamics in the off-gas system.

The issue of the off-gas dynamics due to the headspace and tubing was first addressed by Dekkers [14]. Dekkers [14] modelled the gas phase together as one stirred tank reactor and obtained good results, but without verifying this assumption and neglecting transport delay inherent with tubing. Wu et al. [15] used a black-box approach to identify the relation between OUR and the outlet gases measured by the off-gas analyzer. A drawback is that the parameters in this model do not possess physical relevance and that new experiments are required in each situation to find the proper relation.

Bloemen et al. [13] included the liquid and gas phase to model the oxygen uptake rate. The off-gas system was 
modelled as a series of $n$ ideally stirred tank reactors (CSTR) plus a time delay. The number of CSTR's, their volumes, and other parameters are fitted using identification experiments. Inherently, the identified volumes are nonphysical parameters. The method was designed for highly dynamic pulse response experiments carried out in chemostats, in which the time constants and parameters related to the system, such as $k_{\mathrm{L}} a$ and volume, are approximately constant.

Exact knowledge of $k_{\mathrm{L}} a$ is required in Bloemen's identification approach and a constant volume is maintained during the chemostat cultivations. In contrast to a chemostat a (fed-) batch system is a non-steady-state system with time-varying biomass and volume. Furthermore, instead of the approximately constant $k_{\mathrm{L}} a$ in a chemostat, $k_{\mathrm{L}} a$ is time-varying in (fed-) batch cultivations due to variations in stirrer speed, gas flow rate, broth volume, cells, viscosity, products, etc., and is often poorly known in large- or pilot-scale systems. Also the liquid volume is time-varying and with that the volume of the headspace and the off-gas dynamics are time-varying as well. Finding the proper relation for $k_{\mathrm{L}} a$ in pilot-scale fed-batch cultivation and identifying the off-gas dynamics for all relevant combinations of broth volume, biomass concentration, stirrer speed, etc., would be a difficult and laborious task.

As an alternative, in this work a method for the calculation of OUR from off-gas measurements is proposed by only considering the gas phase and its dynamics. It is based on a white box model containing parameters that are easy to retrieve from the system. The method is therefore applicable without the need for new identification runs. Moreover, the method does not require knowledge of $k_{\mathrm{L}} a$. In fact, by combining off-gas data and measurements of dissolved oxygen, $k_{\mathrm{L}} a$ can be estimated if needed.

In the OUR calculation we are interested in the oxygen fraction at the input of the system, i.e., the auxiliary oxygen fraction entering the headspace, while the measurements take place at the output, i.e., oxygen fraction in the analyzer. The theory and application of the reconstruction of the input using measurements of the output as well as the underlying model are given in the section "Online reconstruction of the inputs and biomass growth". The values of the (system) parameters are given in "Appendix 2". The biomass growth observer and controller are given in "Appendix 1". The new method for OUR computation is an enhancement to the biomass growth observer and controller developed earlier for small-scale laboratory equipment where Eq. 2 is sufficient. Therefore, it is relevant to test the performance of the enhanced system on real experiments in larger scale. This aspect is described in section "Results and discussion". The cultivated biomass is the basis for vaccines currently being developed at the Netherlands Vaccine Institute.

\section{Materials and methods for the experiments}

Experiments for off-gas model validation

The experiments for validation of the model for the off-gas dynamics (Eqs. 5-7a-c) were performed in a 60-1 bioreactor containing 30-401 demineralised water without bacteria. The inlet gas was let into the headspace to mimic the auxiliary oxygen concentration. The inlet gas was excited with known inputs (by changing the gas composition, the gas flow rates, and the volume of the headspace and liquid). The response of the off-gas system (consisting of the headspace, centrifuge, incinerator, and cooler) was recorded every ten seconds (oxygen fraction in the inlet and outlet gases). Oxygen transfer between the liquid and gas phase was assumed negligible.

Pilot-scale cultivation for vaccine production

\section{System set-up}

Figure 1 shows an overview of the bioreactor and measurement system as it was used for pilot-scale vaccine production. The cells were grown in the liquid-phase of the reactor. The gas that leaves the cultivation broth could not be analyzed directly. First it entered the headspace (necessary, e.g., foaming), next the centrifuge (for mechanically defoaming), the incinerator (for killing (pathogenic) microorganisms), the cooler (for drying before the gas can be analyzed) and finally the analyzer. All parts were connected by tubing, which is represented by the tubing box.

The system for monitoring and controlling (fed-) batch cultivations is shown in Fig. 2 [2]. The system consists of:

- Computation of the oxygen uptake rate (OUR) being an essential "measurement" and the starting point for the advanced monitoring and control system.

- Observer for specific growth rate and biomass concentrations. An observer is needed, because there is no sensor available for biomass growth.

- Controller to track the set-point for specific growth rate during the dynamic fed-batch cultivation by manipulating the feed.

- Automatic tuning method to tune the specific growth rate controller automatically without human interference or introduction of excitation signals. Tuning of controllers is necessary to ensure that the process is properly controlled at the desired set-point and does not oscillate or become sluggish due to, e.g., interference with other processes like dissolved oxygen.

- Performance monitor to monitor whether the controller is doing its task well and to decide whether to switch the auto-tuning on or off. 
Fig. 1 Schematic of the bioreactor and measurement system

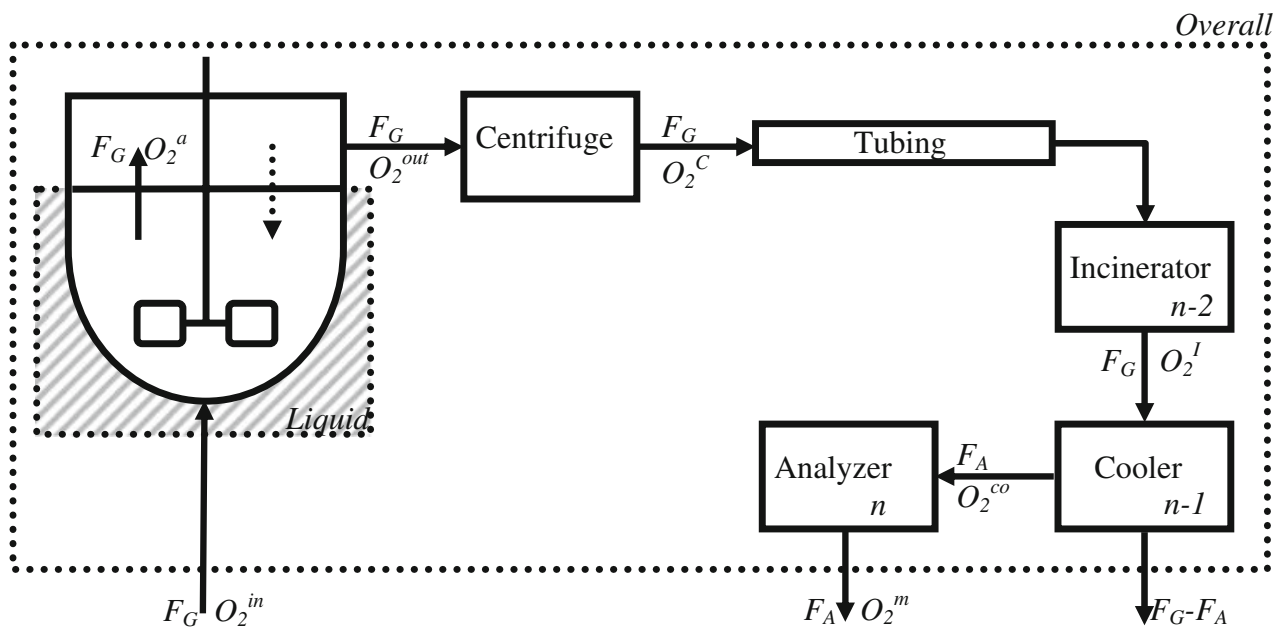

The batch cultivations with $N$. meningitidis were per-

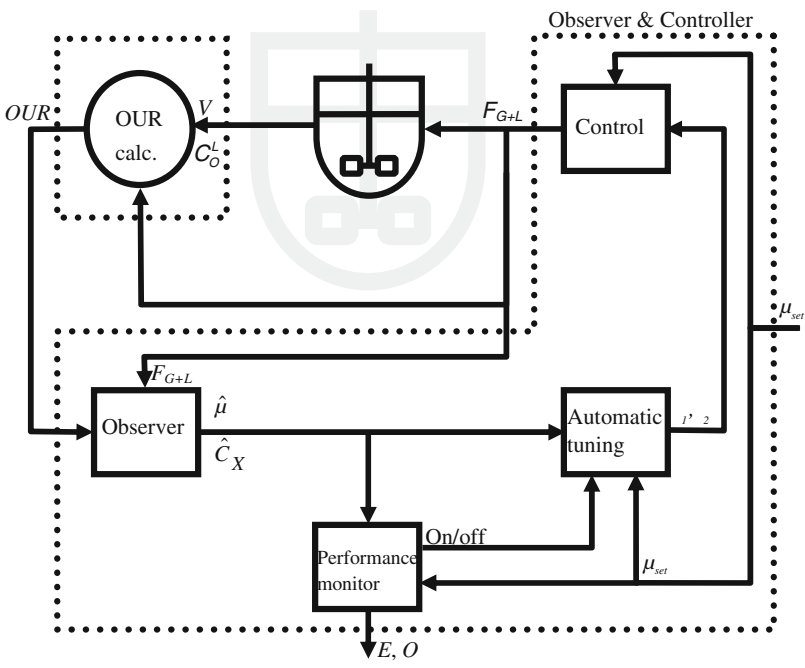

Fig. 2 Schematic monitoring and control system. The dotted boxes indicate the two parts considered for scale-up from laboratory (5 l) to pilot-scale cultivations (60 1): the OUR calculation and the implementation of the observer and controller for biomass growth

\section{Bioreactor conditions and analysis}

Two types of bacteria were grown in the pilot-scale experiments: Neisseria meningitidis and Bordetella pertussis. The oxygen and carbon dioxide fractions in the inlet and outlet gases were measured on a mass spectrometer (prima White Box 600, Thermo Election, UK). The differences in inlet and outlet gas flows were negligible, because the gas production $\left(\mathrm{CO}_{2}\right)$ and consumption $\left(\mathrm{O}_{2}\right)$ rates were approximately equal (respiration quotient is 1 ): $F_{\mathrm{G}} \approx F_{\mathrm{G}}^{\text {in }} \approx F_{\mathrm{G}}^{\text {out }}$. A pressure test was performed in advance of the cultivation. Biomass was measured offline by measuring optical density (OD) at $590 \mathrm{~nm}$ of $1 \mathrm{ml}$ suspension using a Vitalab 10 (Vital Scientific, the Netherlands). Nuclear magnetic resonance NMR (OXFORD NMR AS400, BoveBid, Amsterdam) was applied to measure substrates and metabolites. formed in Baart et al. [12] and were used here for bioreactor monitoring. For detailed materials and methods we refer to [12]. In short, the bacteria were grown in aerobic conditions on a chemically defined medium in a 601 stainless steel bioreactor containing 401 of medium. Dissolved oxygen was controlled by increasing stirrer speed, the difference between inlet and outlet gases by adjusting the gas flow rate via the sparger $\left(1-201 \mathrm{~min}^{-1}\right)$.

The fed-batch cultivations with $B$. pertussis were done to test the monitoring and control system on pilot-scale. The bacteria were grown in aerobic conditions on a chemically defined medium in a 601 stainless steel bioreactor containing 301 of medium at the start. After the batch phase, the fed-batch was started automatically when the limiting substrates were almost depleted and the specific growth rate dropped to the set-point. In the fed-batch phase two 2.51 concentrated stock solutions containing glutamate and lactate were added into the bioreactor to obtain higher biomass concentrations. The specific growth rate was controlled by an adaptive control law ([1,2] and "Appendix 1"). The tuning parameters of the specific growth rate controller $\gamma_{1}$ and $\gamma_{2}$ were adjusted until the performance measures were satisfactory using automatic tuning. Dissolved oxygen was controlled by increasing the stirrer speed. The bioreactor was aerated using headspace aeration $\left(51 \mathrm{~min}^{-1}\right)$.

\section{Online reconstruction of the inputs and biomass growth}

This section consists of three parts:

- The online reconstruction of the inputs using the outputs is given for the generic system in the section "theory".

- In our system, it was not possible to measure the oxygen uptake rate directly, e.g., using a sensor. Consequently it is not possible to verify whether the 
calculated auxiliary oxygen uptake rate fits the real values. The model of the off-gas dynamics that is needed for the reconstruction of OUR, on the other hand, can be verified, because the oxygen fraction can be measured accurately. Indirectly, the accuracy of the off-gas model gives an indication of the accuracy of the calculation of the auxiliary oxygen uptake rate. This model and its validation are given in the section "model off-gas dynamics".

- The application of the theory to the off-gas system of the bioreactor and online reconstruction of biomass growth incorporating the tubing delay are addressed in the section "implementation".

\section{Theory}

In the OUR calculations we are interested in the oxygen fraction at the input of the system, while the measurements take place at the output. Therefore, the aim is to reconstruct the input $u$ from output $y$ (Fig. 3). Suppose a system containing a series of continuous stirred tank reactors. The series of CSTRs are modelled by linear first order systems given by the system matrices $A$ and $B$. The model and system matrices are given in the next section "Model for off-gas dynamics". Equation 3 gives the evolution of the states $x$ along the discrete time axis for the generic system:

$x(t+T)=x(t) \mathrm{e}^{A T}+\int_{\vartheta=t}^{\vartheta=t+T} \mathrm{e}^{A(\vartheta-t)} B u(\vartheta) \mathrm{d} \vartheta$

where $y$ are the outputs, $t$ the time, and $T$ the sampling time. The input $u$ is assumed piecewise constant. The average

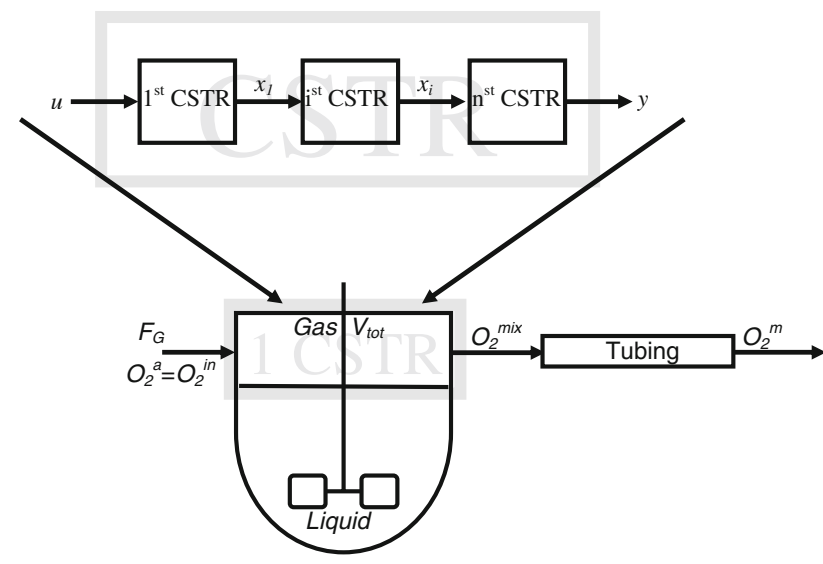

Fig. 3 Schematic of the bioreactor system for modelling the online reconstruction of the input $u$ using output $y$ through a system containing $n$ continuous stirred tank reactors (black) and through a reduced system containing one CSTR (gray). The gas-phase in the reduced model concerns the total gas volume of all compartments $V_{\text {tot }}$. The inlet gas is let into the headspace to mimic the auxiliary oxygen concentration input during $t$ until $t+T(\bar{u}(t))$ is reconstructed by rewriting Eq. 3:

$\bar{u}(t)=\left(A^{-1}\left(\mathrm{e}^{A T}-1\right) B\right)^{-1}\left(x(t+T)-x(t) \mathrm{e}^{A T}\right)$

Model for off-gas dynamics

The dynamics of the off-gas system are modelled by a series of $n$ ideally stirred tanks plus a time delay (back mixing from the headspace to the cultivation broth is assumed negligible) as in [13]:

$$
\begin{aligned}
& \frac{\mathrm{dO}_{2}^{i}}{\mathrm{~d} t}=\frac{F_{\mathrm{G}}}{V^{i}}\left(\mathrm{O}_{2}^{i-1}-\mathrm{O}_{2}^{i}\right), \quad i=1, \ldots, n \\
& \mathrm{O}_{2}^{\mathrm{m}}(t)=\mathrm{O}_{2}^{\mathrm{n}}\left(t-T_{\mathrm{D}}\right)
\end{aligned}
$$

where $\mathrm{O}_{2}^{i}$ is the oxygen fraction in the $i$ th ideally stirred tank (Fig. 1), $\mathrm{O}_{2}^{0}$ is the auxiliary oxygen fraction entering the headspace $\mathrm{O}_{2}^{\mathrm{a}}, F_{\mathrm{G}}$ the gas flow rate, and $V^{i}$ the volume of the gas in the $i$ th ideally stirred tank, which is retrieved from the geometrical properties of the equipment. $T_{\mathrm{D}}$ is the transport delay:

$T_{\mathrm{D}}=\frac{V_{\text {tubing }}}{F_{\mathrm{G}}}$

In contrast to [13] the dynamics are determined on the basis of physical volume measurements of the stirred tanks (centrifuge, incinerator, and cooler) and tubing instead of identification.

A drawback of Eq. 5 concerning the online calculation of OUR is that the signals $\mathrm{O}_{2}^{i}$ must be differentiated $n$ times for the computation of the actual OUR giving more measurement noise. To avoid propagation of the measurement noise a reduced model is proposed in which the gas-phase is considered as one ideally stirred reactor (Fig. 3):

$V_{\mathrm{tot}}=\sum_{i=1}^{n} V^{i}$

$\frac{\mathrm{dO}_{2}^{\operatorname{mix}}}{\mathrm{d} t}=\frac{F_{\mathrm{G}}}{V^{\mathrm{tot}}}\left(\mathrm{O}_{2}^{\mathrm{a}}-\mathrm{O}_{2}^{\mathrm{mix}}\right)$

$\mathrm{O}_{2}^{\mathrm{m}}(t)=\mathrm{O}_{2}^{\mathrm{mix}}\left(t-T_{\mathrm{D}}\right)$

where $V_{\text {tot }}$ is the total volume of the gas phase consisting of the headspace, centrifuge, incinerator, and cooler. $\mathrm{O}_{2}^{\operatorname{mix}}$ is the oxygen concentration after mixing in the total gas phase.

The full model (Eqs. 5-6) and the reduced model (Eqs. 6$7 \mathrm{a}-\mathrm{c})$ were verified by calculating the response of the measurement system to varying $\mathrm{O}_{2}^{\mathrm{a}}, F_{\mathrm{G}}$, and $V_{\mathrm{h}}$ and by comparing the model response with experimental data. In the experiments with water, the inlet gas was let into the headspace to mimic the auxiliary oxygen concentration (Fig. 3). Mass transfer between the liquid and gas phase was neglected.

The oxygen concentration into the headspace $\left(\mathrm{O}_{2}^{\mathrm{a}}\right)$ was varied between 0 and $21 \%$ oxygen and the switching was 

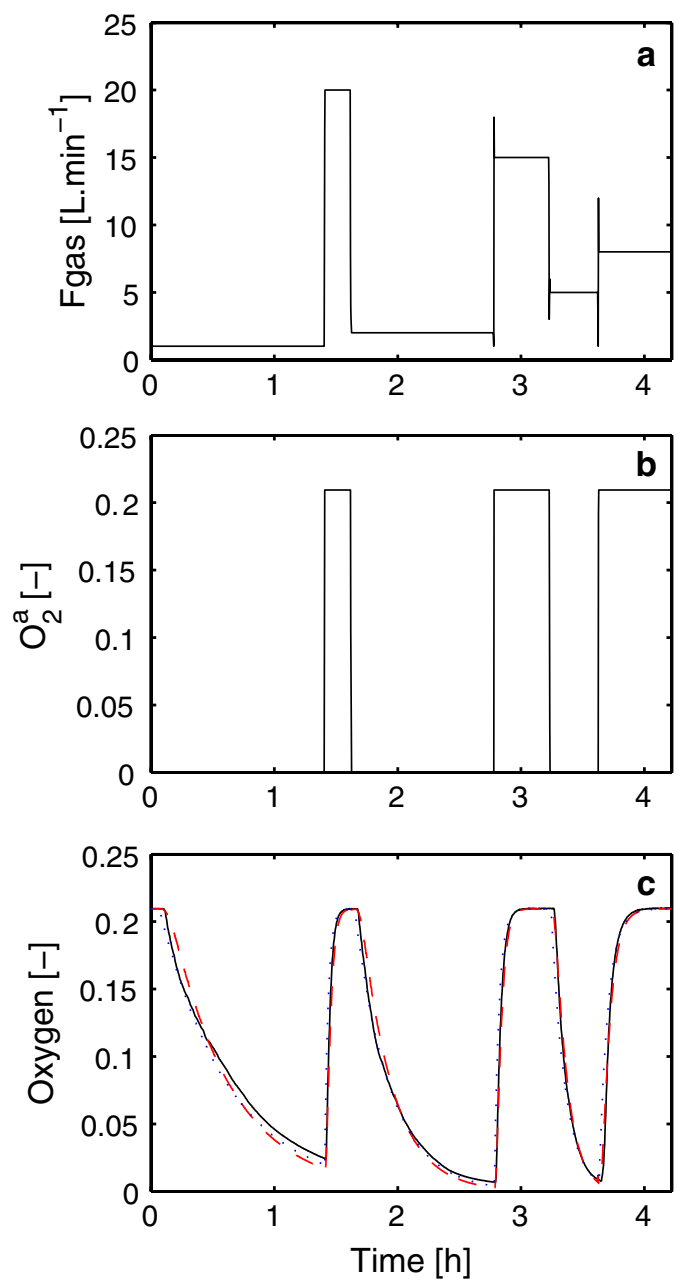

Fig. 4 Dynamics of the gas-phase for a 301 headspace for experimental data. a Gas flow rate. b Auxiliary oxygen fraction. c Measured oxygen fraction (continuous line), oxygen fraction of the full model (four CSTRs plus delay) (red, dashed line), and oxygen fraction of the reduced model (one CSTRs plus delay) (blue, dotted line)

chosen such that the system is allowed to reach steady state at most instances. Figure 4 shows an example of the response of the model and the system for a headspace of 301 . The mean error was $1.1 \times 10^{-3}$ for the full model and $1.3 \times 10^{-3}$ for the reduced model. Both the full model (Eqs. 5-6) and the reduced model (Eqs. 6-7a-c) accurately fit the experimental data.

The reduced model is therefore used in the sequel.

\section{Implementation}

Since the measurements of the oxygen fraction in the outlet stream are delayed due to the tubing and mixing (Figs. 1, 3 ), the following notation is used in the sequel: the subscript $t-T_{\mathrm{D}} \mid t$ denotes the value at $t-T_{\mathrm{D}}$ given all information at $t$. The reconstruction of the input $\mathrm{O}_{2, t-T_{\mathrm{D}} \mid t}^{\mathrm{a}}$ (circle in Fig. 5) is part of the online reconstruction of biomass growth consisting in four steps (Fig. 5).
First, the auxiliary oxygen fraction $\left(\mathrm{O}_{2, t-T_{\mathrm{D}} \mid t}^{\mathrm{a}}\right)$ and auxiliary oxygen uptake rate $\left(\mathrm{OUR}_{t-T_{\mathrm{D}} \mid t}^{\mathrm{a}}\right)$ are calculated to counteract the mixing effects in the gas-phase. Second the observer (EKF) estimates biomass growth $\left(\hat{\mu}_{t-T_{\mathrm{D}} \mid t}\right.$ and $\hat{C}_{X, t-T_{\mathrm{D}} \mid t}$ ). And third, the delay is incorporated by predicting the current biomass concentration based on the delayed observations and the delay itself. The oxygen transfer coefficient can be calculated in a fourth step as an option.

Calculation of $\mathrm{O}_{2, t-T_{\mathrm{D}} \mid t}^{\mathrm{a}}$ and $\mathrm{OUR}_{t-T_{\mathrm{D}} \mid t}^{\mathrm{a}}$. The oxygen uptake rate that does take into account mixing effects is obtained by replacing $\mathrm{O}_{2}^{\mathrm{m}}$ in Eq. 1 by $\mathrm{O}_{2, t-T_{\mathrm{D}} \mid t}^{\mathrm{a}}$ :

$\mathrm{OUR}_{t-T_{\mathrm{D}} \mid t}^{\mathrm{a}} \approx \frac{F_{\mathrm{G}}}{V}\left(\mathrm{O}_{2, t-T_{\mathrm{D}} \mid t}^{\mathrm{in}}-\mathrm{O}_{2, t-T_{\mathrm{D}} \mid t}^{\mathrm{a}}\right) \frac{p}{R T_{\mathrm{L}}}$

The auxiliary oxygen fraction at $t-T_{D}$ given all information at $t \mathrm{O}_{2, t-T_{\mathrm{D}} \mid t}^{\mathrm{a}}$ is reconstructed from online $\mathrm{O}_{2, t-T_{\mathrm{D}} \mid t}^{\mathrm{m}}$ measurements using Eq. 4. To reduce the noise propagation, the reduced model is applied (Eqs. 6-7a-c), in which the gas-phase is considered as one ideally mixed tank reactor. With $x=\mathrm{O}_{2, t-T_{\mathrm{D}} \mid t}^{\mathrm{m}}, \quad u=\mathrm{O}_{2, t-T_{\mathrm{D}} \mid t}^{\mathrm{a}}, \quad A=$ $-\frac{F_{\mathrm{G}, t-T_{\mathrm{D}} \mid t}}{V_{\mathrm{tot}, t-T_{\mathrm{D}} \mid t}}$, and $B=\frac{F_{\mathrm{G}, t-T_{\mathrm{D}} \mid t}}{V_{\mathrm{tot}, t-T_{\mathrm{D}} \mid t}}$ the auxiliary oxygen fraction becomes:

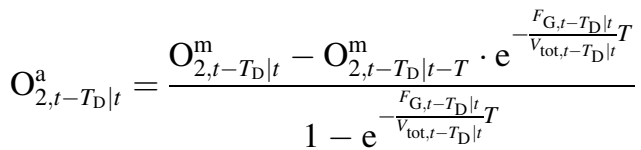

The differences in the measured oxygen signals $\left(\mathrm{O}_{2}^{\mathrm{m}}\right)$ during one time instant $T$ in Eq. 9 are small and therefore noise sensitive. To further reduce the effect of the noise, smoothed signals are calculated using Savitsky-Golay smoothing [16] for a nine point window and a first order polynomial.

Observer for specific growth rate and biomass. An Extended Kalman Filter (EKF) was applied to estimate specific growth rate $\left(\hat{\mu}_{t-T_{\mathrm{D}} \mid t}\right)$ and biomass $\left(\hat{C}_{\mathrm{X}, t-T_{\mathrm{D}} \mid t}\right)$ using the auxiliary oxygen uptake rate $\left(\mathrm{OUR}_{t-T_{\mathrm{D}} \mid t}^{\mathrm{a}}\right)$ every minute [17].

Biomass prediction. The biomass concentration at time $t$ $\left(C_{\mathrm{X}, t \mid t}^{\mathrm{p}}\right)$ was predicted given the delayed information at time $t$ based on exponential growth during the tubing delay $T_{\mathrm{D}}$ (assuming $\mu$ constant during $T_{\mathrm{D}}$ ):

$\mu_{t \mid t}^{\mathrm{p}}=\hat{\mu}_{t-T_{\mathrm{D}} \mid t}$

$C_{\mathrm{X}, t \mid t}^{\mathrm{p}}=\hat{C}_{\mathrm{X}, t-T_{\mathrm{D}} \mid t} \mathrm{e}^{\left(\mu_{t \mid t}^{\mathrm{p}}-\frac{\left.F_{\mathrm{S}, t-T_{\mathrm{D}} \mid t}\right) T_{\mathrm{D}}}{V^{\prime}-T_{\mathrm{D}} \mid t}\right.}$

Computation of oxygen transfer coefficient. The oxygen transfer coefficient $k_{\mathrm{L}} a$ is calculated using the oxygen uptake rate and the measured dissolved oxygen concentration: 
Fig. 5 Schematic of the bioreactor system for online reconstruction of oxygen consumption and growth. The gas-phase includes the gasphase in the bioreactor and all other devices (centrifuge, incinerator, etc.)

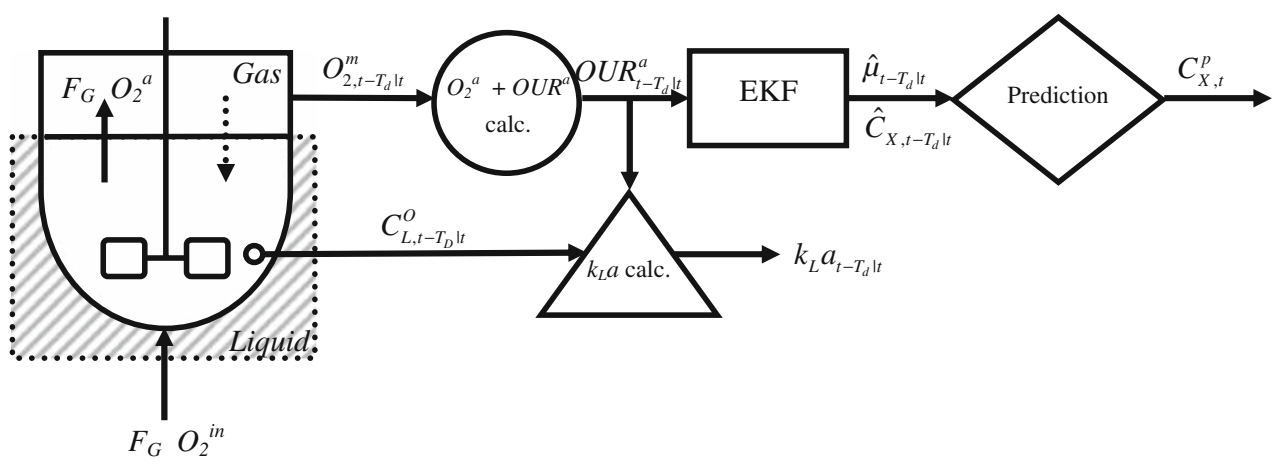

$k_{\mathrm{L}} a_{t-T_{\mathrm{D}} \mid t}=\frac{\mathrm{OUR}_{t-T_{\mathrm{D}} \mid t}^{\mathrm{a}}}{\mathrm{O}_{2, t-T_{\mathrm{D}} \mid t}^{\mathrm{a}} p / H-C_{\mathrm{O}, t-T_{\mathrm{D}} \mid t}^{\mathrm{L}}}$

where $H$ is the Henry coefficient. Note that the oxygen transfer rate is assumed to be equal to the oxygen consumption rate, because dissolved oxygen is controlled and oxygen dynamics are much faster than other relevant processes. To reduce the noise, the signal is again smoothed using Savitsky-Golay smoothing [16] for a nine point window and a first order polynomial.

\section{Results and discussion}

The oxygen uptake rate was calculated using the steady-state method (Eq. 2) and using online reconstruction (Eqs. 8-9). Each of these signals was used as input for the Extended Kalman Filter [17] to observe biomass and specific growth rate and finally to predict biomass concentrations (Eq. 10) to investigate the effect of the off-gas dynamics during real batch and fed-batch experiments. The auto-tuning controller for specific growth rate, developed and validated for smallscale cultivations in $[1,2]$, was applied to the pilot-scale cultivation with $B$. pertussis without any modification.

Eight experiments for $N$. meningitides were evaluated. Figure 6 shows the first five hours of the typical batch cultivation with $N$. meningitidis with sparger aeration. The errors in the calculation of the oxygen uptake rate with the dynamic model (Eqs. 8-9) were up to $30 \%$ smaller than the values calculated from the steady-state method (Eq. 2). As a result the observed biomass concentrations were closer to the samples when the dynamic OUR calculation was used (Eqs. 8-10).

The effect of the off-gas dynamics was significant in the first part of the batch cultivation and decreased with time due to the increasing gas flows and thereby decreasing gas mixing times and transport delays.

Figure 6e shows the typical time-varying course of the oxygen transfer coefficient $k_{\mathrm{L}} a$. In the first part of the cultivation, the cells grew exponentially and so did the demand for oxygen. To cope with this demand, the stirrer speed and gas flow rate increased and as a consequence $k_{\mathrm{L}} a$ increased.
Two B. pertussis cultivations were performed using headspace aeration. The oxygen transfer coefficient was smaller for headspace aeration compared with sparger aeration of the $N$. meningitidis cultivations (compare Fig. 6e with 7c), resulting in smaller differences in inlet and outlet gas concentrations and more noise on the OUR.

The gas flow rate was kept constant $\left(51 \mathrm{~min}^{-1}\right)$ and consequently the mixing effects and tubing delays were approximately constant and small (50 s) compared to the initial phase of the $N$. meningitidis cultivation (4.5 min). As a consequence, the mixing effects and delays in the gas phase were negligible in the experiment with $B$. pertussis due to the higher gas flows applied for headspace aeration.

Figure 7 shows the results for the fed-batch cultivation with $B$. pertussis. First, the cells were grown in batch cultivation until the substrates almost depleted. At $t=18.5 \mathrm{~h}$ the enhancing substrate, lactate, was depleted resulting in a lower specific growth rate; at $19.5 \mathrm{~h}$ the main substrate, glutamate, was depleted and the specific growth rate dropped.

At this point, the feed with limiting substrates and the autotuning controller started automatically to control the specific growth rate at the set-point of $0.06 \mathrm{~h}^{-1}$. At the start of the fedbatch, a few minutes were needed to fill the tubes with the feed and to actually start the substrates entering the bioreactor. After that, as a result of the controller action an exponentially increasing feed was added into the bioreactor to cope with the exponentially increasing biomass. The controller maintained the specific growth rate close to the set-point.

After some hours of fed-batch, the mean error and the oscillation measure (see "Appendix 1" for the definition) decreased and eventually became approximately constant indicating that controller performance was satisfactory and that further auto-tuning would have no effect. The performance monitor qualified these measures to be small and decided to continue the cultivation with the current settings for $\gamma_{1}$ and $\gamma_{2}$ and to deactivate the automatic tuning. This means that the correct tuning has been "learned" after one run. Subsequently, the following cultivations can be performed with the obtained settings.

The observed biomass concentrations coincide well with the offline measurements; and also the specific growth rate 

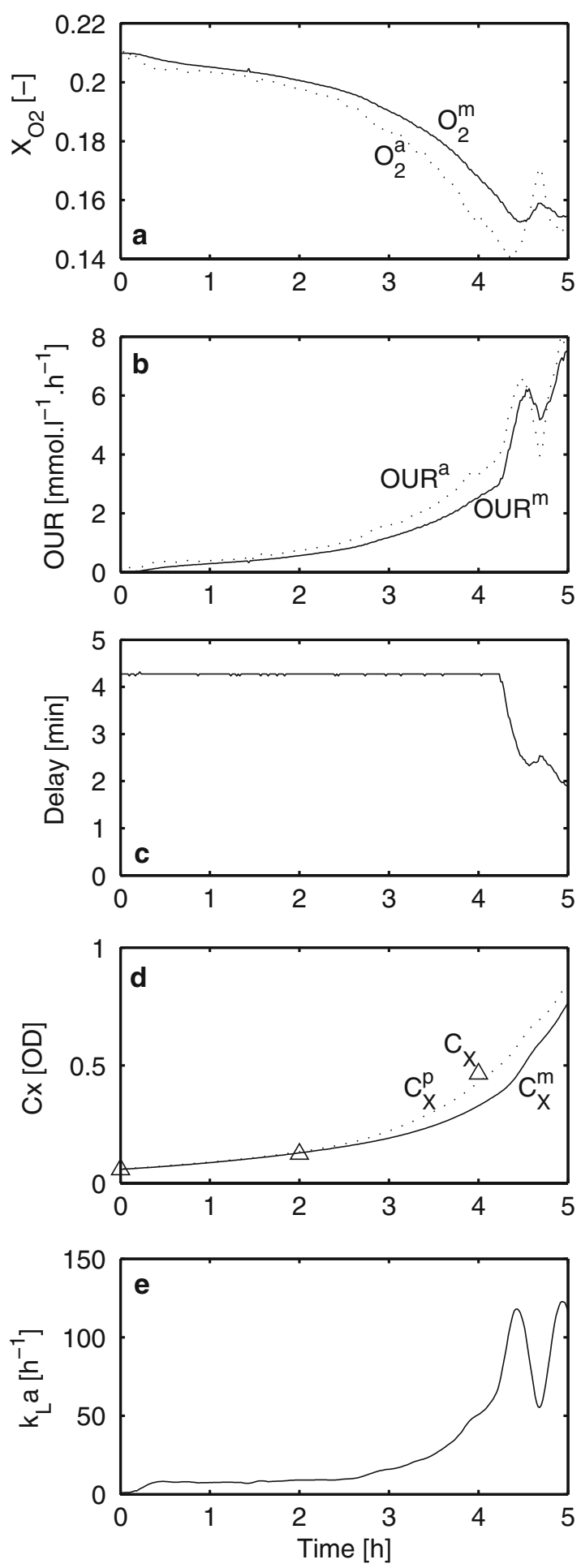

Fig. 6 Experimental results for $N$. meningitidis. a Measured $\left(\mathrm{O}_{2}^{\mathrm{m}}\right)$ and auxiliary oxygen fraction $\mathrm{O}_{2}^{\mathrm{a}}$. b Steady state method $\left(\mathrm{OUR}^{\mathrm{m}}\right)$ and auxiliary oxygen uptake rate $\left(\mathrm{OUR}^{\mathrm{a}}\right)$. c Tubing delay. d Offline biomass measurements (triangles $C_{\mathrm{X}}$ ), biomass observed from OUR ${ }^{\mathrm{m}}$ $\left(C_{\mathrm{X}}^{\mathrm{m}}\right.$, continuous line $)$, and biomass observed and predicted from $\mathrm{OUR}^{\mathrm{a}}\left(C_{\mathrm{X}}^{\mathrm{p}}\right.$, dotted line $)$. e Oxygen transfer coefficient was accurately observed throughout the whole fed-batch cultivation.

Figure $7 \mathrm{~h}$ (left axis) shows that the substrates glutamate and lactate decreased during the batch cultivation and were kept constant at very low concentrations during the fedbatch part of the cultivation. B. pertussis is able to form and excrete acetoacetate in certain conditions, e.g., when glutamate and lactate are depleted, thereby reducing the biomass yield [18]. The undesired component acetoacetate was only formed in small amounts at the start of fed-batch (Fig. $7 \mathrm{~h}$ right axis, note the small values on the scale), when neither glutamate nor lactate were available in the medium. At $t=23 \mathrm{~h}$, glutamate and lactate were again present; the acetoacetate was consumed and remained zero in the following hours of the fed-batch.

Figure $7 \mathrm{c}$ shows the time-varying course of the oxygen transfer coefficient $k_{\mathrm{L}} a$ for $B$. pertussis. The stirrer speed, and so $k_{\mathrm{L}} a$, is increased throughout the cultivation to cope with the increasing oxygen demand. At $t=29 \mathrm{~h}$ the stirrer speed reached its maximum value and remained constant until the end of the cultivation and the dissolved oxygen controller was not capable anymore to track the set-point. The oxygen transfer coefficient, however, decreased due to blanketing effects (caused by, e.g., viscosity, formation of proteins, and lysis).

The applications show that the observer and controller are highly applicable: from small-scale to pilot-scale cultivations; from batch to fed-batch cultivations, from low biomass concentrations to high biomass concentrations, from OUR calculated using the off- gas composition to OUR calculated using $k_{\mathrm{L}} a$, dissolved oxygen and inlet concentrations; and from cultivation with $B$. pertussis to $N$. meningitidis.

\section{Conclusions}

In this work two aspects concerning the scale-up from laboratory to pilot-scale cultivation have been considered and successfully implemented:

- The first is an improved computation of oxygen uptake rate taking into account gas-phase dynamics that is important for large-scale equipment. If the volume of the gas phase is large compared to the gas flows, mixing effects and tubing delays tend to filter out the dynamics of the oxygen uptake rate. Incorporating the gas phase dynamics in online monitoring reduces the error observations of the oxygen consumption and biomass growth up to $30 \%$. Using models which contain system-relevant parameters enhances the applicability of the method. 
Fig. 7 Experimental data for B. pertussis with adaptive control of the specific growth rate. a Measured $\left(\mathrm{O}_{2}^{\mathrm{m}}\right)$ and auxiliary $\left(\mathrm{O}_{2}^{\mathrm{a}}\right)$ oxygen fraction. b Steady-state method (OUR ${ }^{\mathrm{m}}$ ) and auxiliary $\left(\mathrm{OUR}^{\mathrm{a}}\right)$ oxygen uptake rate. c Oxygen transfer coefficient. d Offline $\left(\Delta C_{\mathrm{X}}\right)$ and predicted biomass concentration $\left(C_{\mathrm{X}}^{\mathrm{p}}\right)$ e Specific growth rate. f Substrate feed rate. Glutamate and lactate $\left(F_{\mathrm{G}+\mathrm{L}}\right)$, prior calculation $\left(F_{\text {calc }}\right)$, proportional action $\left(F_{\mathrm{P}}\right)$, and integral action $\left(F_{\mathrm{I}}\right)$. g Controller tuning parameters. h Substrate concentrations: glutamate $\left(C_{\mathrm{G}}\right.$ left axis) and lactate $\left(C_{\mathrm{L}}\right.$ left axis); and acetoacetate ( $C_{\mathrm{A}}$ right axis). i Mean absolute error. j Oscillation measure
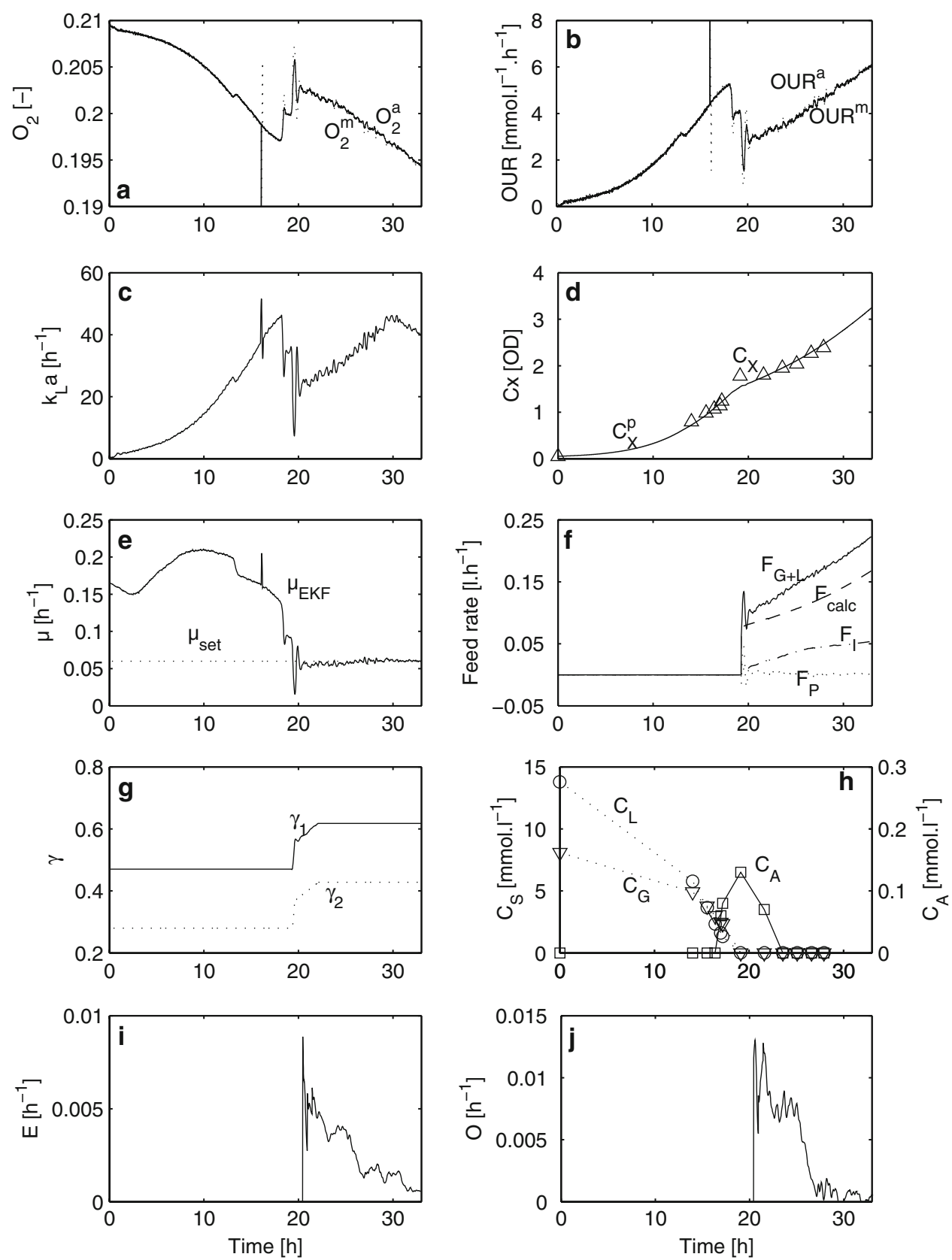

- The second aspect is the monitoring and control of the biomass growth. The monitoring and control systemdesigned for small-scale cultivations-accurately monitored and controlled the specific growth rate and biomass for pilot-scale cultivations in real-time. It appeared that the monitoring and control system could be applied directly to the larger-scale cultivation without modifications like redesigning or re-tuning the observer or controller. So, this strongly suggests that the monitoring and control system is robust with respect to scale and micro-organism.

On large-scale, vaccines are currently produced in batch cultivation. In traditional batch cultivation with $B$. pertussis, the cells grow until the substrates are depleted up to approximately $1.6 \mathrm{OD}$. The methods developed in this paper show that fed-batch cultivation has potential for production on (near) production-scale. In the fed-batch phase of the cultivation the biomass more than doubles up to 3.5 OD by adding a feed with limiting substrates to control the specific growth rate. Higher biomass concentrations can be obtained, like in small-scale cultivations [1], if the headspace aeration were replaced by sparger aeration to obtain proper oxygen transfer.

So, the combination of enhancing an existing process with controlled fed-batch cultivation and the achievement of at least doubled concentration of bulk vaccine on pilot-scale 
yields a favourable upstream production method for bulk whooping cough vaccine.

Acknowledgments Wageningen University and the Netherlands Vaccine Institute (NVI) work together in a project with Applikon Biotechnology BV and Siemens NV in order to improve the production process by release of biopharmaceuticals on the basis of new online techniques. The Dutch Ministry of Economical Affairs (TSGE3067) funds the project. Laboratory experiments were performed at NVI, The Netherlands. We like to thank Martin Hamzink for performing the NMR measurements and Gino Baart for providing the experimental data with $N$. meningitides.

Open Access This article is distributed under the terms of the Creative Commons Attribution Noncommercial License which permits any noncommercial use, distribution, and reproduction in any medium, provided the original author(s) and source are credited.

\section{Appendix 1: Monitoring and control}

The monitoring and control system consists of an observer, controller for specific growth rate, auto-tuning of the controller, and a performance monitor.

Observer. An Extended Kalman Filter (EKF) was applied to estimate specific growth rate $\left(\mu^{\mathrm{m}}\right)$ and biomass $\left(C_{\mathrm{X}}^{\mathrm{m}}\right)$ using the oxygen uptake rate (OUR) every minute [17]. The EKF is based on the following model for biomass growth and oxygen consumption:

$\begin{aligned} \frac{\mathrm{d} C_{\mathrm{X}}}{\mathrm{d} t} & =\mu C_{\mathrm{X}} \\ \text { OUR } & =\left(\frac{\mu}{Y_{\mathrm{O}}}+m_{\mathrm{O}}\right) C_{\mathrm{X}}\end{aligned}$

Specific growth rate control. The control purpose is to regulate the specific growth rate $(\mu)$ to a desired value by adding a feed with limiting substrates using the controller of [1]:

$F_{\mathrm{G}+\mathrm{L}}=F_{\text {calc }}+F_{\mathrm{P}}+F_{\mathrm{I}}$

$F_{\text {calc }}=\frac{a c+b d}{a C_{\mathrm{G}}^{\mathrm{in}}+b C_{\mathrm{L}}^{\text {in }}} C_{\mathrm{X}}^{\mathrm{p}} V$

$F_{\mathrm{P}}=K_{\mathrm{P}} \varepsilon$

$F_{\mathrm{I}}=\int_{0}^{t} K_{\mathrm{I}} \varepsilon \mathrm{d} t$

where the error $\varepsilon$ is the set point minus the observed specific growth rate $\left(\mu_{\mathrm{set}}-\mu_{\mathrm{p}}\right)$ and where the controller gains $K_{\mathrm{P}}$ and $K_{\mathrm{I}}$ are adjusted online to the changing volume:

$K_{\mathrm{P}}=\frac{V}{\gamma_{1}\left(a C_{\mathrm{G}}^{\text {in }}+b C_{\mathrm{L}}^{\text {in }}\right)}, \quad K_{\mathrm{I}}=\frac{V}{\gamma_{2}\left(a C_{\mathrm{G}}^{\text {in }}+b C_{\mathrm{L}}^{\text {in }}\right)}$

$\gamma_{1}$ and $\gamma_{2}$ are the tuning parameters for the controller and $a$, $b, c$, and $d$ are constants depending on the micro-organism (in the work $B$. pertussis) and the set-point for specific growth rate. Automatic tuning was performed as in [2]: $\frac{\mathrm{d} \gamma_{1}}{\mathrm{~d} t}=\beta_{1} \frac{\varepsilon}{\mu_{\mathrm{set}}}+\beta_{2} \frac{\varepsilon^{2}}{\mu_{\mathrm{set}}^{2}}$

$\frac{d \gamma_{2}}{d t}=\beta_{1} \frac{\varepsilon}{\mu_{\mathrm{set}}}+\beta_{2} \frac{\varepsilon^{2}}{\mu_{\mathrm{set}}^{2}}$

where the tuning parameters $\gamma_{1}$ and $\gamma_{2}$ are adapted proportional to the error $\varepsilon$ and proportional to the quadratic error $\varepsilon^{2}$, which is large if the error is large. $\beta$ is the adaptation rate, the tuning parameters for auto-tuning.

Performance monitor. The performance monitor has two tasks in the system. The first function of the performance monitor is to qualify controller performance using the criteria given in Eqs. 15-16 [2]. Second, based on the qualification, the performance monitor decides to retune the process using automatic tuning or to stop the autotuning activity and continue with the current settings. The first measure is the mean absolute error over the past $N$ points of a moving window at time point $k$ :

$E(k)=\frac{1}{N} \sum_{k-N}^{k}|\varepsilon(k)|$

The second is the oscillation measure and is defined by

$O(k)=\frac{1}{N}\left(\sum_{k-N}^{k}|\varepsilon(k)|-\sum_{k-N}^{k} \varepsilon(k)\right)$

\section{Appendix 2: Parameter values Table 1}

Table 1 Parameter values

\begin{tabular}{ll}
\hline Parameter & Value \\
\hline Volume (1) & \\
Headspace & $20-30$ \\
Centrifuge & 1.34 \\
Tubing & 2.0 \\
Incinerator & 2.15 \\
Cooler & 0.66 \\
Cultivation broth & $30-40$ \\
$B$. pertussis & \\
$Y_{\mathrm{O}}$ & $0.033 \mathrm{OD} \mathrm{mmol}^{-1}$ \\
$m_{\mathrm{O}}$ & $0.69 \mathrm{mmol} \mathrm{OD}^{-1} \mathrm{~h}^{-1}$ \\
$N$. meningitidis & \\
$Y_{\mathrm{O}}$ & $0.077 \mathrm{OD} \mathrm{mmol}^{-1}$ \\
$m_{\mathrm{O}}$ & $0 \mathrm{mmol} \mathrm{OD}^{-1} \mathrm{~h}^{-1}$ \\
$C_{\mathrm{L}}^{\text {in }}$ & $835 \mathrm{mmol} \mathrm{l}^{-1}$ \\
$C_{\mathrm{G}}^{\text {in }}$ & $500 \mathrm{mmol} \mathrm{l}^{-1}$ \\
$\tau_{\text {analyzer }}$ & $13 \mathrm{~s}^{-1}$ \\
$\mu_{\text {set }}$ & $0.06 \mathrm{~h}^{-1}$ \\
$\beta_{1}$ & 0.25 \\
$\beta_{2}$ & 0.50 \\
$N$ & 90 \\
\hline &
\end{tabular}




\section{References}

1. Soons ZITA, Voogt JA, Van Straten G, Van Boxtel AJB (2006) Constant specific growth rate in fed-batch cultivation of Bordetella pertussis using adaptive control. J Biotechnol 125:252-268. doi:10.1016/j.jbiotec.2006.03.005

2. Soons ZITA, Voogt JA, Van Straten G, Van der Pol LA, Van Boxtel AJB (2007) Online automatic tuning and control for fedbatch cultivation. Bioprocess Biosyst Eng online first. doi: 10.1007/s00449-007-0182-4

3. Wang NS, Stephanopoulos GN (1984) Computer applications to fermentation processes. CRC Crit Rev Biotechnol 2:1-90

4. Casas López JL, Rodríguez Porcel EM, Oller Alberola I, Ballesteros Martin MM, Sánchez Pérez JA, Fernández Sevilla JM, Chisti Y (2006) Simultaneous determination of oxygen consumption rate and volumetric oxygen transfer coefficient in pneumatically agitated bioreactors. Ind Eng Chem Res 45:11671171. doi:10.1021/ie050782a

5. Estler MU (1995) Recursive on-line estimation of the specific growth rate from off-gas analysis for the adaptive control of fedbatch processes. Bioprocess Eng 12:205-207. doi:10.1007/ BF01767468

6. Levisauskas D (2001) Inferential control of the specific growth rate in fed-batch cultivation processes. Biotechnol Letters 23:1189-1195. doi:10.1023/A:1010528915228

7. Siegell SD, Gaden EL Jr (1962) Automatic control of dissolved oxygen levels in fermentation. Biotechnol Bioeng 4:354-356. doi:10.1002/bit.260040311

8. Tatiraju S, Soroush M, Mutharasan R (1999) Multi-rate nonlinear state and parameters estimation in a bioreactor. Biotechnol Bioeng 63:22-32

9. Spriet JA, Botterman J, De Buyser DR, De Visser PL, Vandamme EJ (1982) A computer-aided noninterfering on-line technique for monitoring oxygen-transfer characteristics during fermentation processes. Biotechnol Bioeng 24:1605-1621. doi:10.1002/ bit.260240713
10. Lubenova VN (1999) Stable adaptive algorithm for simultaneous estimation of time-varying parameters and state variables in aerobic bioprocesses. Bioprocess Biosyst Eng 21:219-226. doi: $10.1007 / \mathrm{s} 004490050667$

11. Ghoul M, Boudrant J, Engasser JM (1991) A comparison of different techniques for the control of the growth of Candida utilis CBS 621. Proc Biochem 26:135-142. doi:10.1016/ 0032-9592(91)80009-E

12. Baart GJE, De Jong G, Philippi M, Van't Riet K, Van Der Pol LA, Beuvery EC, Tramper J, Martens DE (2007) Scale-up for bulk production of vaccine against meningococcal disease. Vaccine 25:6399-6408. doi:10.1016/j.vaccine.2007.06.008

13. Bloemen HHJ, Wu L, Gulik WM, Heijnen JJ, Verhaegen MHG (2003) Reconstruction of the $\mathrm{O}_{2}$ uptake rate and $\mathrm{CO}_{2}$ evolution on a time scale of seconds. Bioeng Food Natural Products 49:1895-1908. doi:10.1002/aic.690490725

14. Dekkers RM (1982) State estimation of a fed-batch baker's yeast fermentation, modelling and control of biotechnological processes. Helsinki, Finland, published by Perfamom, Oxford, pp 201-211

15. Wu L, Lange HC, Gulik WM, Heijnen JJ (2003) Determination of in vivo oxygen uptake and carbon dioxide evolution rates from off-gas measurements under highly dynamic conditions. Biotechnol Bioeng 81:448-458. doi:0.1002/bit.10480

16. Gorry PA (1990) General least-squares smoothing and differentiation by the convolution (Savitzky-Golay) method. Anal Chem 62:570-573. doi:10.1021/ac00205a007

17. Soons ZITA, Shi J, Stigter JD, Van der Pol LA, Van Straten G, Van Boxtel AJB (2008) Observer design and tuning for biomass growth and kLa estimation using online and offline measurements. J Process Contr 18:621-631. doi:10.1016/j.jprocont. 2007.12.008

18. Thalen M, Van den Ijssel J, Jiskoot W, Zomer B, Roholl P, De Gooijer C, Beuvery C, Tramper H (1999) Rational medium design for Bordetella pertussis; basic metabolism. J Biotechnol 75:147-159. doi:10.1016/S0168-1656(99)00155-8 